\title{
Avaliação da armadilha HP iscada com diferentes taxas de liberação de octenol na captura de anofelinos (Diptera: Culicidae) em Brejo do Mutambal, Município de Varzelândia, Estado de Minas Gerais
}

\author{
Evaluation of the HP trap baited with different release rates of octenol for \\ capturing anophelines (Diptera: Culicidae) in Brejo do Mutambal, \\ municipality of Varzelândia, State of Minas Gerais
}

\section{Renata Antonaci Gama ${ }^{1}$, Andrey José de Andrade ${ }^{1}$, Mateus Ramos de Andrade ${ }^{1}$, Marcelo Carvalho de Resende ${ }^{2}$ e Álvaro Eduardo Eiras ${ }^{1}$}

\begin{abstract}
RESUMO
Para avaliar o papel do octenol na coleta de anofelinos foram utilizadas armadilhas HP iscadas com diferentes taxas de evaporação. Foram capturados 285 anofelinos e não houve diferença entre o número de anofelinos coletados com as armadilhas controle e as iscadas com octenol e nem entre as taxas de evaporação.
\end{abstract}

Palavras-chaves: Anopheles spp. Cairomônio. Octenol. Armadilhas HP.

\begin{abstract}
To evaluate the role of octenol in collecting anophelines, HP traps baited with different rates of evaporation. A total of 285 anophelines was captured and there was no significant difference in the numbers of anophelines captured between the control traps and the traps baited with octenol and no difference between the release rates.
\end{abstract}

Key-words: Anopheles spp. Kairomone. Octenol. HP traps.

0 gênero Anopheles Meigen 1818 conta atualmente com aproximadamente 517 espécies distribuídas nas regiões tropicais e temperadas do mundo, das quais 54 são encontradas no Brasil e 70 incriminadas como transmissoras de plasmódios da malária humana $a^{14}$.

O Estado de Minas Gerais, apesar de não ser considerado atualmente como uma área de elevada transmissão de malária, já 0 foi no passado ${ }^{1}$ e ainda possui relatos de transmissão ${ }^{3}$. A fauna de anofelinos no Estado é elevada e documentada em algumas localidades perfazendo aproximadamente 27 espécies $^{12}$.

No presente, ainda não se dispõe de uma armadilha específica e eficiente para a captura e conseqüente monitoramento de anofelinos. Existem diversos dispositivos utilizados como armadilha para a coleta de mosquitos, dentre esses as armadilhas CDC e Shannon são as mais conhecidas e utilizadas ${ }^{10} .0$ acréscimo de atraentes sintéticos específicos facilitaria e efetivaria as coletas de anofelinos, uma vez que o principal método de coleta é realizado com isca humana expondo o coletor ao risco de se infectar por diferentes agentes etiológicos como plasmódios e arbovírus.

o 1-octen-3-ol (octenol) é um cairomônio identificado por Hall a partir de substâncias voláteis emanadas pela expiração de bovinos ${ }^{13}$, mas sua atratividade para fêmeas de mosquitos é bastante questionada, embora se observe que armadilhas iscadas com 0 cairomônio resultam na atração de mosquitos em campo ${ }^{14} 18 \mathrm{e} \mathrm{em}$ laboratório5.

0 objetivo desse trabalho foi avaliar a armadilha HP iscada com diferentes taxas de liberação do octenol para a coleta de anofelinos na vila de Brejo do Mutambal (MG).

\footnotetext{
1. Laboratório de Culicídeos, Departamento de Parasitologia, da Universidade Federal De Minas Gerais, Belo Horizonte, MG. 2. Núcleo de Entomologia, Fundação Nacional de Saúde, Belo Horizonte, MG

Endereço para correspondência: Dra ${ }^{\mathrm{a}}$ Renata Antonaci Gama. Av. Antônio Carlos 6627, 31270-901 Belo Horizonte, MG.

Telefax: 5531 3499-2834/2970

e-mail: antonaci@icb.ufmg.br

Recebido em: 26/09/2006

Aceito em: 20/06/2007
} 


\section{MATERIAL E MÉTODOS}

Local de coleta. Os experimentos de campo foram realizados durante o mês de abril (2005) no município de Varzelândia, MG na vila de Brejo do Mutambal (15033"13 S; 4400"18W). O lugarejo foi reconhecido como vila em 1990 e sua população está estimada em 2.049 pessoas, Fallin Grain Genomics, INC: http://www.fallingrain.com/ world/BR/15/Brejo do Mutambal.html, Acesso em: 20/02/2006 .

Armadilha utilizada. As armadilhas luminosas $\mathrm{HP}^{17}$ foram instaladas no peridomicílio e em borda de mata, sendo penduradas em árvores com sua parte mais baixa do corpo de PVC a aproximadamente $1,50 \mathrm{~m}$ do solo. As mesmas foram instaladas às 18:00h e recolhidas às 6:00h do dia seguinte.

Dose resposta do octenol. Foram comparadas quatro taxas de evaporação do octenol, obtidas em laboratório ${ }^{21}(0,5$; 5; 15 e 30mg/h), como atraentes nas armadilhas luminosas. Como controle foi utilizada a armadilha luminosa (sem octenol). Os cinco tratamentos foram dispostos seguindo o delineamento Quadrado Latino 5 X 5, totalizando cinco repetições por taxa de evaporação do octenol/noite e, conseqüentemente, 25 armadilhas/ noite. Duas áreas distintas foram escolhidas para a instalação das armadilhas, uma área de borda de mata (dois quadrados) localizada próxima a uma lagoa e constituída por vegetação abundante, e outra de peridomicílio (três quadrados) abrangendo o quintal das residências, que na maioria das vezes apresentava algum tipo de criação, principalmente de cães e galinhas.

Acondicionamento dos exemplares coletados. Os anofelinos capturados foram transferidos para envelopes entomológicos, etiquetados com o número da armadilha, data da coleta e números de indivíduos capturados para posteriormente serem identificados no Laboratório de Culicídeos, Instituto de Ciências Biológicas da Universidade Federal de Minas Gerais ${ }^{4}$.

Análise estatística. Foi utilizado o teste não-paramétrico de Kuskal-Wallis para comparação entre as taxas de liberação de octenol, e o número total de capturas de cada biota (área de borda de mata e peridomicílio) foi comparado pelo do teste nãoparamétrico de Mann-Whitney. Todos os cálculos foram realizados no programa estatístico SYSTAT ${ }^{\circledR}$ Versão 8.0.

\section{RESULTADOS}

Durante todo o experimento foi coletado um total de 285 anofelinos distribuídos em seis espécies (Tabela 1) sendo que a maior parte do material coletado não pode ser identificado até espécie e nem até subgênero devido às más condições em que os insetos se encontravam, sendo os mesmos identificados até gênero. Dos 285 anofelinos coletados, 97,2\% foram fêmeas e os machos presentes na amostra $(2,8 \%)$ não puderam ser identificados.

Todos os indivíduos identificados pertenciam ao subgênero Nyssorhynchus e as espécies encontradas foram: Anopheles benarrochi, Anopheles darlingi, Anopheles albitarsis sp, Anopheles oswaldoi, Anopheles argyritarsis e Anopheles triannulatus (Tabela 1). A média de anofelinos capturados em
Tabela 1 - Total e porcentagem de exemplares de cada espécie de anofelinos coletados em área de peridomicílio e mata na vila de Brejo do Mutambal, município de Varzelândia (MG) em abril de 2005.

\begin{tabular}{|c|c|c|c|c|c|c|}
\hline \multirow[b]{3}{*}{ Espécie } & \multicolumn{4}{|c|}{ Local } & & \\
\hline & \multicolumn{2}{|c|}{ peridomicílio } & \multicolumn{2}{|c|}{ mata } & \multicolumn{2}{|c|}{ Total } \\
\hline & $\mathrm{n}^{0}$ & $\%$ & $\mathrm{n}^{\mathrm{O}}$ & $\%$ & $\mathrm{n}^{0}$ & $\%$ \\
\hline Anopheles benarrochi & 14 & 17,1 & 27 & 13,3 & 41 & 14,4 \\
\hline Anopheles argyritarsis & 9 & 11,0 & 5 & 2,5 & 14 & 4,9 \\
\hline Anopheles albitarsis & 2 & 2,4 & 9 & 4,4 & 11 & 3,9 \\
\hline Anopheles darlingi & 1 & 1,2 & 3 & 1,5 & 4 & 1,4 \\
\hline Anopheles oswaldo $i^{*}$ & 1 & 1,2 & 3 & 1,5 & 4 & 1,4 \\
\hline Anopheles triannulatus & 0 & 0,0 & 1 & 0,5 & 1 & 0,3 \\
\hline Anopheles spp & 55 & 67,1 & 155 & 76,3 & 210 & 73,7 \\
\hline Total & 82 & 28,8 & 203 & 71,2 & 285 & 100,0 \\
\hline
\end{tabular}

* ou Anopheles konderi

área de borda de mata para armadilhas iscadas foi maior com octenol liberado na taxa de $15 \mathrm{mg} / \mathrm{h}(15,4 \pm 7,3)$ e na área de peridomicílio a melhor captura foi obtida com a taxa de $0,5 \mathrm{mg} / \mathrm{h}$ $(4,6 \pm 4,4)$ (Figura 1). A menor quantidade anofelinos capturados em área de borda de mata foi obtida com a taxa de $5 \mathrm{mg} / \mathrm{h}$ $(4,2 \pm 2,1)$ e no peridomicílio com $15 \mathrm{mg} / \mathrm{h}(0,6 \pm 0,6)$. Apesar de ter sido coletado maior número de anofelinos nas armadilhas iscadas com octenol e esse aumento parecer ser dose dependente (aumentando a resposta com o aumento da taxa de liberação) essa diferença não foi estatisticamente significativa, nem entre as taxas de liberação e nem entre as armadilhas controle e iscadas $(\mathrm{p}>0,05)$.

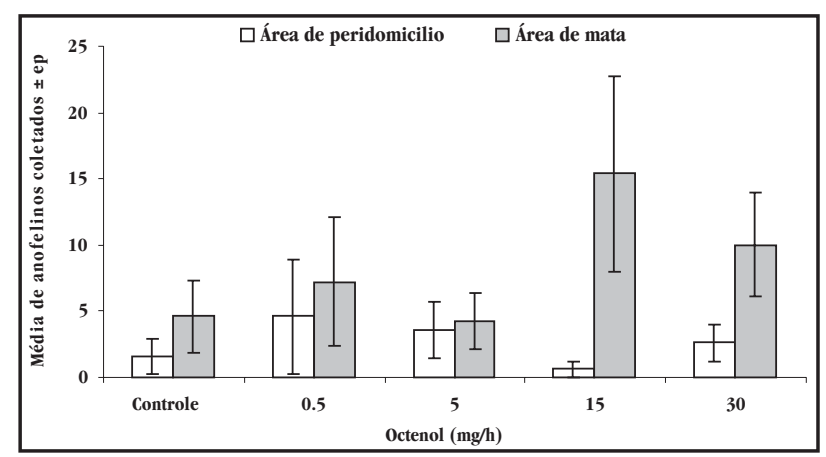

Figura 1 - Comparação entre a média de anofelinos coletados em armadilhas HP contendo diferentes taxas de liberação de octenol em área de peridomicílio e mata na Vila de Brejo do Mutambal, município de Varzelândia (MG), em abril de 2005 (Kruskal-Wallis, p> 0,05).

\section{DISCUSSÃo}

As espécies coletadas em Brejo do Mutambal já foram relatadas no Estado de Minas Gerais ${ }^{12912} 15$. Devido ao mau estado de conservação do material não foi possível identificar todos os exemplares coletados sendo, portanto a fauna de Brejo do Mutambal provavelmente mais rica em espécies do que foi observado.

A utilização de armadilhas na captura de anofelinos é interessante no que se refere à proteção dos coletores principalmente em áreas de alta transmissão de malária humana, entretanto até o presente momento ainda não se obteve resultados 
promissores em comparação à isca humana, exceto para 0 mosquito Aedes aegypti ${ }^{13}$. Uma das armadilhas bastante utilizada é a do tipo CDC cuja fonte atrativa é a luz sendo por isso uma armadilha generalista. Outro problema dessa armadilha é que grande parte dos insetos coletados podem ser danificados como aconteceu neste trabalho com $73,7 \%$ dos exemplares.

Para potencializar as capturas de insetos hematófagos com a armadilha do tipo CDC, pode-se acrescentar atraentes sintéticos, como $\mathrm{CO}_{2}$ e o octenol ${ }^{1618192021}$. O $\mathrm{CO}_{2}$ apesar de ser um atraente bastante utilizado e eficiente, é muito generalista, pois a maioria dos insetos hematófagos o utiliza para localização dos hospedeiros ${ }^{6}$.

0 octenol vem sendo empregado em diversos trabalhos para a coleta de mosquitos visando sua utilização como atraente e/ou sinergista em armadilhas, entretanto sua eficiência ainda é questionada. Palis ${ }^{16}$ na Cidade de Maracay (México) ao comparar a armadilha CDC com lâmpada UV acrescida de diferentes atraentes para captura de Anopheles albimanus e Anopheles aquasalis observou que, para a primeira espécie, o octenol potencializou significativamente a captura quando comparado a armadilha com apenas a luz (712 exemplares para o octenol e 356 para o controle). Vythilingam e cols ${ }^{21}$ ao instalarem armadilhas CDC iscadas com octenol não observaram diferenças significativas na coleta de Anopheles maculatus quando comparado a CDC só com a luz. Entretanto, os exemplares coletados foram em número insignificante nos dois tratamentos $(0 \pm 0$ para o primeiro tratamento e $0,89 \pm 0,89$ para o segundo).

No presente trabalho realizado em Brejo do Mutambal também não foi observado um papel sinérgico do octenol com a luz na coleta de anofelinos em armadilhas HP, não obstante tenha se observado elevada captura dos mesmos. Apesar do octenol no não ter aumentado significativamente a captura de anofelinos, a taxa de $15 \mathrm{mg} / \mathrm{h}$ parece ser a mais indicada para captura de mosquitos em área com maior cobertura vegetal. Provavelmente, a armadilha luminosa HP não é a mais apropriada para 0 acréscimo de atraentes. A possível adequação e utilização de uma outra armadilha poderiam ser mais eficazes do que a HP, como por exemplo, a armadilha BG-Trap ${ }^{\otimes 7} 11$ que por produzir uma pluma de odor semelhante a liberada pelo ser humano, poderia ser acrescida do cairomônio octenol e ser eficaz na coleta desses insetos como vem sendo na coleta de outros culicídeos como Aedes e Culex? .

\section{AGRADECIMENTOS}

Ao Dr. Edelberto Santos Dias (CPqRR/FIOCRUZ - MG) pelo apoio e suporte no trabalho de campo.

\section{REFERÊNCIAS}

1. Amaral ADF, Penido, HM. Distribuição dos anofelinos no vale do Rio Doce. Revista do Serviço Especial de Saúde Pública 1:163-172, 1947.

2. Andrade RM, Leal JM. Distribuição de anofelinos na bacia hidrográfica do rio São Francisco. Estados de Minas Gerais, Bahia, Goiás, Pernambuco, Alagoas e Sergipe. Revista Brasileira de Malariologia 1:147-163, 1960.
3. Chaves KM, Zumpano JF, Resende MC, Junior FP, Rocha MOC. Malária em Minas Gerais, Brasil, no período 1980-1992. Cadernos de Saúde Pública 11:621-626, 1995.

4. Consoli RAGB, Oliveira RL. Principais mosquitos de importância sanitária no Brasil, Rio de Janeiro, 1994.

5. Cork A, Park KC. Identification of electrophysiologically-active compounds for the malaria mosquito, Anopheles gambiae, in human sweat extracts. Medical and Veterinary Entomology 10:269-276, 1996.

6. Eiras AE. Mediadores químicos entre hospedeiros e insetos vetores de doenças médico-veterinárias. In: Vilela EF, Della Lucia TMC (eds) Feromônios de insetos: biologia, química e emprego no manejo de pragas. Editora Holos, Ribeirão Preto, capítulo 12, p. 99-122, 2001.

7. Eiras AE, Geier M. Depósito de Patente. WO 2004/034-783A2. 29 de abril de 2004.

8. Fallin Rain Genomics, Inc. http://www.fallingrain.com.world/BR/15/Brejo do Mutambal.html. Acesso em 20/02/2006.

9. Ferrete JA, Lemos JC, Lima SC. Estudo da fauna de anofelinos em áreas rurais e periurbanas do município de Uberlândia - MG. In: III Simpósio regional de geografia: "Perspectivas para o cerrado no século XXI". Universidade Federal de Uberlândia, Uberlândia, p. 1-12, 2003.

10. Forattini OP. Culicidologia Médica, Identificação, biologia e epidemiologia. Editora da Universidade de São Paulo, 2002

11. Freitas RM, Eiras AE, Oliveira RL. Field evaluation of effectiveness of the BGSentinel, a new trap for capturing adult Aedes aegypti (Diptera: Culicidae). Memórias do Instituto Oswaldo Cruz 101:321-325, 2006.

12. Guedes AS, Freitas JR. Contribuição ao conhecimento da distribuição geográfica dos anofelinos e algumas observações sobre a biologia do Anopheles darlingi Root, 1926, no Estado de Minas Gerais, Brasil. Revista Brasileira de Malariologia 5:157-165, 1953 .

13. Hall DR, Beevor PS, Cork A, Nesbitt BF, Vale GA. A potent olfactory stimulant and attractant for tsetse isolated from cattle odours. Insect Science an its application 5:335-339, 1984 .

14. Lozovei AL. Culicídeos. In: Marcondes CB (ed) Entomologia Médica e Veterinária. Editora Atheneu, São Paulo, p. 59-103, 2001.

15. Maciel CS. Lista de culicineos do Estado de Minas Gerais, Brasil (Diptera, Culicidae). Revista Brasileira de Malariologia e Doenças Tropicais 14:465-494, 1962.

16. Palis YR. Evaluation of ligth traps combined with carbon dioxide and 1-octen3-ol to collect anofelines in Venezuela. American Mosquito Control Association 12:91-96, 1996.

17. Pugedo H, Barata RA, França-Silva JC, Silva JC, Dias ES. HP: um modelo apropriado de armadilha luminosa de sucção para a captura de pequenos insetos. Revista da Sociedade Brasileira de Medicina Tropical 38:70-72, 2005.

18. Silva IM. Avaliação de componentes sintéticos do odor humano como atraentes para Aedes aegypti (Diptera: Culicidae) em laboratório e campo. Tese (Doutorado em Parasitologia). Universidade Federal de Minas Gerais, Belo Horizonte, 2003.

19. Takken W, Kline, D.L. Carbon dioxide and 1-octen-3-ol as mosquitoes attractants. Journal American Mosquito Control Association 5:311-316, 1989.

20. Van Den Hurk AF, Beebe NW, Ritchie SS. Responses of mosquitoes of the Anopheles faurati complex to 1-octen-3-ol and light in combination with carbon dioxide in northen Queensland, Australia. Medical Veterinary Entomological 11:177-180, 1997.

21. Vythilingam I, Lian CG, Thim CS. Evaluation of carbon dioxide and 1-octen-3-ol as mosquito attractants. Southeast Asian Journal Tropical Medical Public Health 23:328-331, 1992. 